\title{
The development of a web-based brief alcohol intervention in reducing heavy drinking among college students: an Intervention Mapping approach
}

\author{
CARMEN V. VOOGT ${ }^{*}$, EVELIEN A. P. POELEN ${ }^{1}$, \\ MARLOES KLEINJAN ${ }^{1}$, LEX A. C. J. LEMMERS ${ }^{2}$ \\ and RUTGER C. M. E. ENGELS ${ }^{1}$ \\ ${ }^{1}$ Behavioural Science Institute, Radboud University Nijmegen, P.O. Box 9104, 6500 HE Nijmegen, The \\ Netherlands and ${ }^{2}$ Trimbos Institute, Netherlands Institute of Mental Health and Addiction, P.O. Box 725, \\ 3500 AS Utrecht, The Netherlands \\ *Corresponding author. E-mail: c.voogt@bsi.ru.nl
}

\section{SUMMARY}

In the Netherlands, young adults' drinking practices have become an issue of public concern since their drinking levels are high. Heavy drinking can place young adults at an increased risk for developing shortand long-term health-related problems. Current national alcohol prevention programmes focus mainly on adolescents and their parents and paying less systematic attention to young adults. The present study describes the theory and evidence-based development of a web-based brief alcohol intervention entitled What Do You Drink (WDYD). We applied the Intervention Mapping (IM) protocol to combine theory and evidence in the development and implementation of WDYD. The WDYD intervention aims to detect and reduce heavy drinking of young adults who are willing to decrease their alcohol consumption, preferably below the Dutch guidelines of low-risk drinking. According to the IM protocol, the development of WDYD resulted in a structured intervention. Reducing heavy drinking to low-risk drinking was proposed as the behavioural outcome. Motivational interviewing principles and parts of the I-Change Model were used as methods in the development of WDYD, whereas computer tailoring was selected as main strategy. An effect and a process evaluation of the intervention will be conducted. IM was found to be a practical instrument for developing the WDYD intervention tailored to a specific target population in the area of alcohol prevention.

Key words: heavy drinking; college students; Intervention Mapping; web-based brief alcohol intervention

\section{INTRODUCTION}

Alcohol use among young adults is recognized as a major public health problem in most Western countries with high social and economic costs (Rehm et al., 2009). The Netherlands has a drinking culture in which individuals start drinking regularly at a relatively young age compared with other Western countries and show relatively fast progress to heavy drinking patterns during the transition from adolescence to young adulthood (McKinnon et al., 2003; Hibell et al., 2004, 2009; Verster, 2011; Ji et al., 2012). Heavy drinking can place young adults, especially college students and those who are affiliated with fraternities and sororities (Maalsté, 2000; Ragsdale et al., 2011; Hallett et al., 2012), at an increased risk for developing short- and 
long-term health-related consequences, including risky sexual behaviour (Hingson et al., 2003), brain damage (Zeigler et al., 2005), problematic alcohol use in adulthood (O'Neill et al., 2001), liver damage (Norstrom and Ramstedt, 2005) and various types of cancer (Rehm et al., 2009).

The heavy drinking practices of Dutch young adults urgently demand adequate interventions that would encourage them to change their alcohol consumption. Current national alcohol prevention programmes concentrate mainly on adolescents in secondary education and their parents. Most adolescents who are younger than 18 years of age follow secondary education and are relatively easy to reach at school. However, after adolescents turn 18, the prevalence rates of alcohol use increase substantially (Poelen et al., 2005). Moreover, at this age, they can legally purchase light beverages ( $\geq 16$ years) and strong alcoholic beverages ( $\geq 18$ years). Ironically, less systematic attention to prevention is given to this important age group in the Netherlands. This is partly due to the increasing difficulty to reach young adults through schools. Nonetheless, there is a clear need for alcohol prevention for young adults aged 18-24 years old.

The present study describes the theory and evidence-based development of the What Do You Drink (WDYD) web-based brief alcohol intervention for Dutch heavy drinking young adults aged 18-24 years old who are motivated to change their drinking behaviour. Our definition of heavy drinking is based on measures of heavy alcohol use and binge drinking. Heavy alcohol use is defined as a mean consumption rate of more than 14 or 21 glasses of standard alcohol units per week for females and males, respectively (Gezondheidsraad, 2006). Binge drinking is defined as drinking five or more glasses of standard alcohol units on one drinking occasion at least once per week (Hibell et al., 2004).

The Intervention Mapping (IM) protocol is a stepwise approach to describe the planned process for theory and evidence-based development, implementation and evaluation of health promotion interventions (Bartholomew et al., 2001). IM elaborates on the fifth phase of the PRECEDE-PROCEED model (Green and Kreuter, 1991), but it is not a theoretical framework by itself, and it has not yet been evaluated or compared with other health promotion planning frameworks (Fernández et al., 2005).
Web-based brief alcohol interventions developed on the basis of theory and evidence are limited. This makes it difficult to identify the effective characteristics of the intervention, to relate effective characteristics to effect sizes (Webb et al., 2010), or to replicate the interventions in different situations (Kwak et al., 2006). Studies that have documented the development of web-based brief alcohol interventions mainly describe the theoretical basis, structure and/or content of intervention (Hallett et al., 2009; Linke et al., 2011). However, these studies do not describe the planned process for the development, implementation and evaluation of these types of interventions, while a planned procedure can improve the chance of success (Brug et al., 2005). The detailed description of the planned process for the development, implementation and evaluation of an intervention, which is unique to IM, has incremental value above these similar approaches. Therefore, the first version of the IM protocol was applied using five steps. IM has proved to be a practical instrument in the development of interventions aimed at nutrition (Reinaerts et al., 2008), weight gain prevention (Kwak et al., 2006), sun protection (Tripp et al., 2000), HIV and pregnancy prevention (Tortelo et al., 2005) and cervical cancer screening (Fernández et al., 2005). This is the first study to use IM to develop an intervention in the field of alcohol prevention.

\section{NEED ASSESSMENT}

Prior to the beginning of the IM process, it is essential to conduct a need assessment (NA) to identify the health problem, its behavioural risk factors and their associated individual and environmental determinants for the at-risk target population. The end product of the NA is the desired behavioural outcome, which should be defined in terms of desired reduction in the health problem. The current study identified heavy drinking among Dutch college students aged 18-24 years old as the health problem. The most important literature on determinants related to heavy drinking among young adults aged 18-24 years old are presented in the next sections.

Several individual and environmental determinants are related to heavy drinking [e.g. (Ham and Hope, 2003)]. Previous research has demonstrated that social norms are related to 
Table 1: Proximal programme objectives specified per determinant

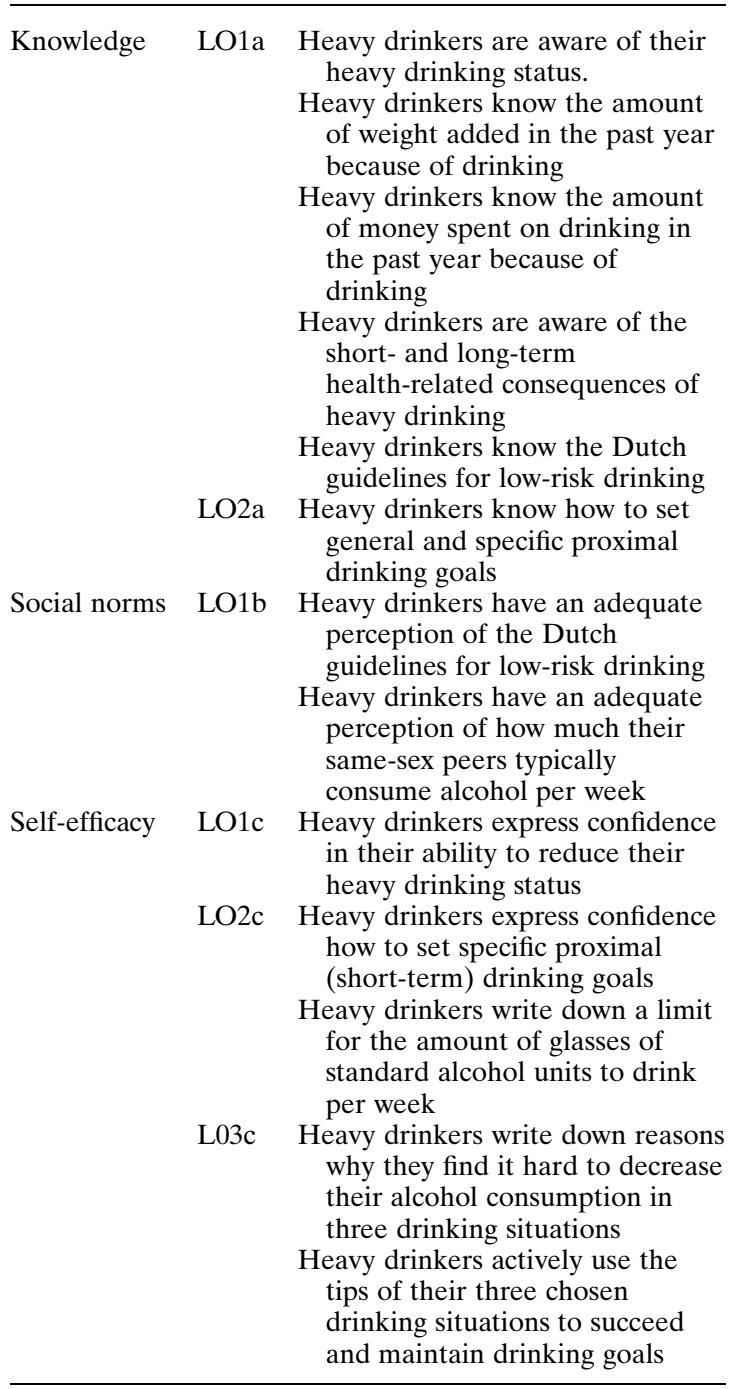

heavy drinking among college students in the age range of 17-25 years old [e.g. (Cullum et al., 2010; Danielsson et al., 2010)]. It is well established, both cross-sectionally [e.g. (Wood et al., 2001)] and longitudinally [e.g. (Danielsson et al., 2010)] that young adults who report higher descriptive norms of alcohol use are more likely to engage in heavy drinking themselves. Although cross-sectional and longitudinal associations between descriptive norms and heavy drinking have been found, associations between injunctive norms and heavy drinking are more limited and less consistent, possibly due to the subjective nature of injunctive norms (Lewis et al., 2010). Therefore, brief alcohol interventions utilize mainly descriptive norms instead of injunctive norms [e.g. (Doumas et al., 2009; Turrisi et al., 2009)].

A substantial number of studies have shown that alcohol expectancies are related to heavy drinking (Greenbaum et al., 2005). Alcohol expectancies refer to beliefs about the positive or negative cognitive, affective or behavioural effects of alcohol (Borsari et al., 2007). Positive expectations (e.g. relaxation) represent an important component of motivation to drink, whereas negative expectations (e.g. cognitive impairment) represent an important component of motivation to restrain (Jones et al., 2001). The more positive one's alcohol expectations, the more heavily one drinks and the greater the likelihood of engaging in heavy drinking (Oei and Morawska, 2004). Alcohol expectancies appeared to be particularly important among adolescents who have yet to decide to drink. Nonetheless, on the other hand, drinking motives appeared to be more important than did alcohol expectancies (Anderson et al., 2009).

Attitudes toward drinking have also been considered found to be related to heavy drinking [e.g. (Ham and Hope, 2003)]. Research has indicated that young adults who perceive low risk and high benefits of alcohol are more likely to consume alcohol (Lundborg and Lindgren, 2002). Contrary, young adults are less likely to engage in heavy drinking when they have positive attitudes and expectancies towards naturally occurring alcohol-free activities, such as studying (Murphy et al., 2007). Increasing knowledge about the health consequences of alcohol can help young adults perceive risks associated with alcohol consumption more accurately (Lundborg and Lindgren, 2002).

Drinking refusal self-efficacy is identified as final determinant of heavy drinking. Young adults with low self-efficacy to avoid heavy drinking in social situations are more likely to engage in heavy drinking [e.g. (Oei et al., 2005; Young et al., 2006)], whereas high drinking refusal self-efficacy predicts lower drinking quantity and frequency, as well as experiencing less problems (Collins and Carey, 2007).

The NA resulted in the following desired behaviour change, which is defined as the behavioural outcome for the purpose of this intervention: 'Dutch college students aged 18-24 
years old who are motivated to change their drinking behaviour in the near future will drink within the normative limits of the Dutch National Health Council for low-risk drinking. This implies that their consumption will not exceed a mean heavy alcohol use consumption of more than 14 (females) or 21 (men) glasses of standard alcohol units per week and/or, in case of binge drinking, five or more glasses of standard alcohol units on one drinking occasion at least once per week within 1 month and 6 months after the intervention'.

\section{INTERVENTION MAPPING}

\section{Step 1: Definition of proximal programme objectives}

Step 1 of the IM process identifies the proximal programme objectives (PPOs), providing the foundation for the intervention by specifying who and what will change because of the intervention. First, the behavioural outcome is divided into performance objectives (POs) by identifying and selecting changeable individual and environmental determinants that are associated with the health problem. Subsequently, the theories and models of behaviour change are used to assess the changeability of the selected determinants. The POs and the determinants are combined in a matrix of PPOs (see Table 1). Finally, the learning objectives (LOs), defined as statements of what the target population should learn to change in order to achieve the desired behavioural outcome (Bartholomew et al., 2001), are formulated.

The behaviour outcome is subdivided into the following POs: deciding to reduce heavy drinking, setting drinking goals and maintaining drinking goals. Knowledge, social norms and self-efficacy are identified as most important and changeable intervention components that should be addressed. Knowledge, although not sufficient in itself, is a prerequisite for determinant of intentional behaviour change (Van Empelen et al., 2003). Social norms is another prerequisite for behavioural change (Bandura, 1986) and seems to be relevant for deciding to reduce heavy drinking and setting drinking goals. Self-efficacy is expected to be the final prerequisite for behavioural change (Kok et al., 1991) and seems to be relevant to all the POs.

\section{Step 2: Selection of intervention methods and strategies}

Step 2 of the IM process links the PPOs, theorybased methods and practical strategies for changing health behaviours.

A substantial body of evidence indicates that brief alcohol interventions can effectively reduce heavy drinking among young adults and students [e.g. (Bewick et al., 2008, Kypri et al., 2009)]. Brief alcohol interventions are timelimited preventive consultations that focus on early detection of problematic alcohol use and motivation of nontreatment-seeking heavy drinkers to change their behaviour or seek treatment (Spijkerman et al., 2010). These types of interventions are based on motivational interviewing (MI) principles (Miller and Rollnick, 2002) and social influence models (Bandura, 1986). MI refers to 'a client-centred, directive method for enhancing intrinsic motivation to change by exploring and resolving ambivalence' (Miller and Rollnick, 2002) and includes goalsetting and action planning components (Bodenheimer and Handley, 2009). The presentation of discrepant personal information to increase one's motivation to change or modify his or her drinking behaviour is generally a central component of brief alcohol interventions (Spijkerman et al., 2010). Therefore, most brief alcohol interventions consist of two parts (i) a screening procedure and (ii) personalized feedback based on the screening outcomes. Topics that are addressed in the screening and feedback include personal drinking profile (e.g. quantity-frequency consumed), risk factors (e.g. negative consequences) and normative comparisons (e.g. perceptions about peers' drinking). The inclusion of normative feedback is based on models on social influence processes (Bandura, 1986) and refers, in this case, to the presentation of comparative information about personal drinking levels and drinking levels of a relevant comparison group, such as same-sex peers (Spijkerman et al., 2010). The provision of personalized feedback, based on the individuals' personal situation, implies that the intervention is 'tailored'. According to the literature, tailored interventions are more effective compared with general interventions because the receiver of the intervention identifies him or herself with personally relevant information. In addition, the receiver pays more attention to the message because the tailored intervention contains more 
relevant and less redundant information compared with general interventions (De Vries and Brug, 1999a).

Originally, brief alcohol interventions were delivered by face-to-face methods (Borsari and Carey, 2000) and postal mail methods (Wild et al., 2007). Recently, interventions are also delivered electronically via computer programmes (Neighbors et al., 2004) and Internet (Kypri et al., 2009). This web-based approach has certain advantages over the more conventional delivery methods. (i) Young adults prefer electronic feedback compared with face-to-face feedback (Kypri et al., 2003), (ii) it allows easy access to large audiences, (iii) it allows participants to access the intervention at their own convenience, (iv) the brief character of the intervention is time efficient and easier to implement and (v) tailored information can be offered in an automated, cost-effective and flexible way (Riper et al., 2009). Web-based brief alcohol interventions may be particularly suitable for college students, especially considering that the majority of young adults in Western countries have access to the Internet and make frequent use of Internet technologies (Lebo, 2010). Therefore, computer tailoring was used to tailor the intervention to each participant.

MI principles and parts of the I-Change model (De Vries et al., 2003) were used as methods to develop WDYD, whereas computer tailoring was selected as main strategy. The I-Change model or the Integrated Model is derived from the Attitude-Social Influence-Self-efficacy (ASE) model (De Vries and Brug, 1999b) that integrates aspects of several theories including the Theory of Planned Behavior (Ajzen, 1991), the Social Cognitive Theory (Bandura, 1986), the Transtheoretical Model (Prochaska and Velicer, 1997) and the Health Belief Model (Janz and Becker, 1984) in order to explain behavioural change process. The I-Change model assumes that at least three phases, awareness, motivation and action, can be distinguished in the behavioural change process. For each phase, different determinants are relevant. Our target population is heavy drinking young adults aged 18-24 years old who are motivated to change their drinking behaviour and therefore are thought to move into the action phase. Hence, WDYD focuses predominantly on the action phase of the behaviour change process with knowledge, social norms and self-efficacy as key determinants (see Figure 1).

\section{Step 3: Programme plan}

Step 3 of the IM process translates the intervention methods and strategies into the programme plan. The intervention components and intervention materials are developed and produced as part of the programme plan in order to conduct and implement a pilot study among representatives of the target population to pre-test the intervention.

Three steps were undertaken for the development of WDYD. First, the chosen strategy was operationalized into a programme plan. Second, representatives of the Trimbos Institute designed the programme materials of WDYD. The Trimbos Institute is the National Institute of Mental Health and Addiction in the Netherlands. Goals of the Trimbos Institute are (i) enhancing the quality of life by engaging in the development and application of knowledge about mental health and addiction and (ii) undertaking evidence-based activities intended to contribute to and facilitate changes in mental health and addiction care to stimulate individual health gains, promote more effective treatment methods and provide models for more efficient care. Finally, WDYD was pre-tested with the target population on user friendliness, design and ideas, and adapted, when necessary, to produce the final version.

WDYD aims to detect and reduce heavy drinking of young adults who are willing to decrease their alcohol consumption, preferably below the Dutch guidelines of low-risk drinking. The first part of WDYD focuses on increasing participants' awareness of the potential problems, consequences, and risks associated with their drinking behaviour based on MI principles. It contains (i) a homepage, (ii) a screening test addressing participants' name, sex, age, education level, weight, alcohol use, willingness to change alcohol consumption, average expenses on one alcohol beverage and descriptive social norms and (iii) personalized feedback that is tailored to participants' sex, alcohol intake and perceived social norms.

The WDYD homepage provides information about who the intervention targets (i.e. young adults who drink alcohol frequently), what you receive from the intervention (i.e. personalized feedback) and the duration to complete the intervention (i.e. about $20 \mathrm{~min}$ ). After the homepage, the intervention proceeds with the screening test. The personalized feedback from the 


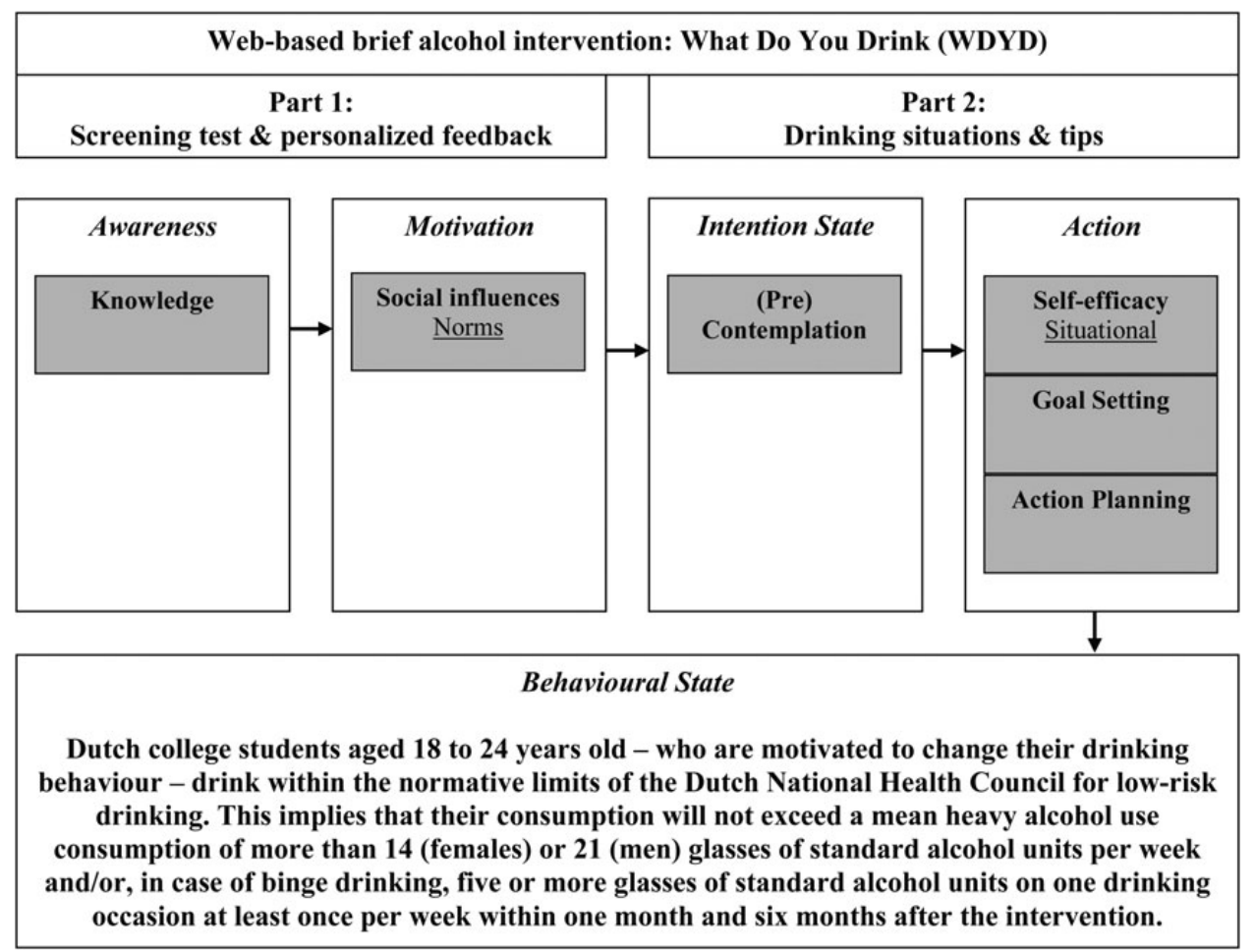

Fig. 1: I-Change Model applied for the web-based brief alcohol intervention WDYD.

screening test includes (i) advice about drinking according to the guidelines of the Dutch National Health Council (Gezondheidsraad, 2006), (ii) the amount of glasses of standard alcohol units that the participant consumed in the last year, with estimates of the number of calories consumed, the amount of weight added because of drinking and the amount of money spent on drinking and (iii) a bar chart comparing the number of glasses of standard alcohol units per week that participants think their same-sex peers consume with the number of glasses of standard alcohol units per week that participants' same-sex peers actually consume. The comparative data of the descriptive norms from same-sex peers are retrieved from alcohol prevalence estimates obtained from the same-sex nationally representative sample of the general population (CBS, 2010). The tone of voice of the personalized feedback was non-judgemental and reflective conform MI principles.

The second part of WDYD focuses on setting and maintaining drinking goals, with a general goal of reducing heavy drinking. Participants are prompted to make decisions about the maximum amount of glasses of standard units of alcohol they want to drink every day of the week at a given time point. Setting such specific proximal (short-term) goals, also called action plans, are found to be more effective than setting distal (long-term) goals (Bodenheimer and Handley, 2009). After the sign-in procedure through a unique login and security identification code, participants are offered an overview of the amount of glasses of standard alcohol units they typically consume per week based on their answers to the questions on the screening test. Subsequently, participants operationalized action plans about the maximum amount of glasses of standard alcohol units they want to drink every day of the week, preferably within the limits of low-risk drinking. Besides goalsetting and action planning, WDYD focuses on strengthening participants' drinking refusal selfefficacy (Oei et al., 2005) to succeed and maintain drinking goals.

After participants have operationalized their action plans, they are asked to choose 3 out of the 12 provided drinking situations in which they find it hard to resist alcohol. Focus group 
Table 2: Overview of 26 drinking situations

\begin{tabular}{|c|c|c|c|}
\hline Drinking situations & $M$ & $S D$ & Range \\
\hline 1. When I am at a party ${ }^{\mathrm{a}, \mathrm{b}, \mathrm{c}}$ & 3.28 & 0.72 & $1.33-5.33$ \\
\hline 2. When my friends are drinking ${ }^{a}$ & 3.18 & 0.70 & $1.33-5.00$ \\
\hline $\begin{array}{l}\text { 3. When I am at the Greek letter } \\
\text { organization }^{\mathrm{a}, \mathrm{b}}\end{array}$ & 2.94 & 0.73 & $1.00-5.33$ \\
\hline 4. When I am free the next day ${ }^{a, b}$ & 2.93 & 0.62 & $1.00-5.00$ \\
\hline $\begin{array}{l}\text { 5. When I am at a nightclub/ } \\
\text { concert }^{\text {a,c }}\end{array}$ & 2.89 & 0.79 & $1.00-5.00$ \\
\hline $\begin{array}{l}\text { 6. When someone offers me a } \\
\text { drink }^{\mathrm{a}}\end{array}$ & 2.87 & 0.65 & $1.00-4.67$ \\
\hline 7. When I am watching $\mathrm{TV}^{\mathrm{a}}$ & 2.83 & 0.45 & $1.00-4.00$ \\
\hline 8. When I am dining out ${ }^{\mathrm{a}}$ & 2.81 & 0.52 & $1.33-4.33$ \\
\hline $\begin{array}{l}\text { 9. When my boy/girlfriend is } \\
\text { drinking }^{\mathrm{a}}\end{array}$ & 2.80 & 0.58 & $1.00-4.33$ \\
\hline $\begin{array}{l}\text { 10. When I have already paid for } \\
\text { my drinks }\end{array}$ & 2.79 & 0.86 & $1.00-5.00$ \\
\hline $\begin{array}{l}\text { 11. When I have finished my } \\
\text { exams }^{\text {a,b }}\end{array}$ & 2.71 & 0.77 & $1.00-5.33$ \\
\hline $\begin{array}{l}\text { 12. When I have just finished } \\
\text { playing sport } \mathrm{a}^{\mathrm{a} b}\end{array}$ & 2.69 & 0.44 & $1.00-4.33$ \\
\hline 13. When I am by myself & 2.66 & 0.30 & $1.00-3.67$ \\
\hline 14. When I am at a festival ${ }^{\mathrm{a}, \mathrm{b}}$ & 2.65 & 0.82 & $1.00-4.67$ \\
\hline $\begin{array}{l}\text { 15. When I am listening to music } \\
\text { or reading }\end{array}$ & 2.64 & 0.31 & $1.00-4.00$ \\
\hline $\begin{array}{l}\text { 16. When I participate in a café } \\
\text { sport (e.g. darts, pool })^{\mathrm{b}}\end{array}$ & 2.64 & 0.55 & $1.00-4.67$ \\
\hline 17. When I first arrive home & 2.63 & 0.25 & $1.00-3.67$ \\
\hline $\begin{array}{l}\text { 18. When I am on the way home } \\
\text { from school }\end{array}$ & 2.61 & 0.31 & $1.00-3.33$ \\
\hline 19. When I feel nervous & 2.61 & 0.27 & $1.00-3.67$ \\
\hline 20. When I am $\mathrm{l}$ & 2.61 & 0.34 & $1.00-4.00$ \\
\hline 21. When I am worried & 2.60 & 0.24 & $1.00-3.00$ \\
\hline 22. When I feel upset & 2.60 & 0.25 & $1.00-3.33$ \\
\hline 23. When I feel sad & 2.60 & 0.30 & $1.00-3.67$ \\
\hline 24. When I feel frust & 2.58 & 0.29 & $1.00-3.33$ \\
\hline 25. When I am feelin & 2.58 & 0.27 & $1.00-3.33$ \\
\hline 26. When I am angry & 2.57 & 0.29 & $1.00-3.67$ \\
\hline
\end{tabular}

Note. $n=158$.

${ }^{\text {a The }} 12$ selected drinking situations for WDYD. The scores of frequency, intensity and self-efficacy were summed to get one mean score of the drinking situations, with higher scores reflecting higher frequency and intensity and lower self-efficacy.

${ }^{\mathrm{b}}$ Drinking situations retrieved from focus group discussions.

c'When I am at a party' and 'When I am at a nightclub/ concert' were taken together into one drinking situation 'When I am going out (party/nightclub/concert)'.

discussions were conducted with the target population to identify these drinking situations. Seven different drinking situations were identified and added to the 19 drinking situations of the revised adolescents' version of Young's drinking refusal self-efficacy questionnaire [DRSEQ-RA: (Oei et al., 2005, Young et al., 2007)]. Subsequently, these 26 drinking situations were examined quantitatively online using a sample of heavy drinking young adults $(n=$ 158) in order to select drinking situations for WDYD. Assessed were (i) the frequency of drinking one or more glasses of standard alcohol units on each drinking situation in the previous week, (ii) the number of glasses of standard alcohol units they had drunk on each drinking situation in the previous week and (iii) the respondents' ability to resist drinking alcohol in each drinking situation. In total, a set of 12 drinking situations was selected for WDYD (see Table 2). After selecting 3 out of the 12 provided drinking situations (e.g. 'When my friends are drinking', 'When I am dining out', 'When someone offers me a drink'), participants are asked to give a rationale why they find it hard to resist alcohol in these drinking situations. Finally, each of the chosen drinking situations provides several tips to resist alcohol and cope with these situations in order to succeed and maintain the general and specific drinking goals.

\section{Step 4: Adaptation and implementation plan}

Step 4 of the IM process focuses on adoption and implementation of the intervention. The end product is a plan for adoption and implementation a programme that would influence behaviour of individuals who will make decisions about adopting and using the programme.

The collaboration with the Trimbos Institute provides a high potential to ensure effective distribution of information and an adequate large-scale implementation, since WDYD could be easily incorporated in their materials and programmes. Practitioners in the field of alcohol prevention and health promotion will further be informed about the effectiveness of WDYD through a large-scale mailing distributed to interested organizations and institutions. Depending on its effectiveness, organizations (e.g. STAP, Dutch Institute for Alcohol Policy) will be encouraged to make referrals to WDYD through their official websites and in other alcohol prevention communications. Therefore, we will send out promotion materials about WDYD to all interested parties. Furthermore, if effective, WDYD will be incorporated into materials of the national mass media campaign for youth alcohol prevention, the national campaign and programme on alcohol and parenting, and the 'Healthy School and Drugs' (Gezonde School en Genotmiddelen) and I.Com (Innovation Centre 
of Mental Health \& Technology) programmes, both run by the Trimbos Institute. Since the tested intervention involves a web-based brief alcohol intervention, it will be easy to implement by providing the WDYD link on websites or in promotion and education materials. Some specific actions for further implementation will consist of adding the WDYD link to promotion materials and on the official website of the national youth alcohol prevention campaign 'Mainstage'. Within the framework of this mass media campaign, youth-oriented activities and contests that will be organized to create publicity will promote WDYD. In addition, WDYD will be integrated in prevention materials that are developed for students and in online modules for the national school prevention programme 'Healthy School and Drugs'. Finally, another relevant direction for further implementation will be the integration of WDYD in more extensive e-health modules developed at I.Com.

\section{Step 5: Evaluation plan}

Step 5 of the IM process entails an effect and a process evaluation of the intervention. The primary objective of the effect evaluation is to test the effectiveness of WDYD. To determine the effectiveness of WDYD, a two-arm parallel group randomized controlled trial will be conducted among 908 heavy drinking college students (for other evaluations of web-based brief interventions, see Riper et al., 2008 and Wallace et al., 2011). One month before the intervention, participants in both the experimental $(n=$ 454: web-based brief alcohol intervention) and control condition ( $n=454$ : no intervention) will receive five weekly repeated online Ecological Momentary Assessment (EMA) measurements (Stone and Shiffman, 1994). After these five measurements, participants in the experimental condition will be exposed to WDYD. Immediately after the intervention, participants in both conditions will receive an EMA measurement of alcohol-related cognitions, that is, attitudes, self-efficacy, subjective norms and alcohol expectancies. One week after the intervention, participants will receive weekly EMA measurements for 6 months and a final EMA measurement 9 months after the intervention. Both in the pre-tests and post-tests, EMA will be used to assess participants' alcohol use and alcohol-related cognitions (Voogt et al., 2011).
The process evaluation - which concerns how and why the intervention was effective or not (Steckler and Linnan, 2002)-will be assessed by (i) the percentage of participants that participated in the intervention, (ii) evaluating which intervention components were actually delivered to the participants, (iii) asking participants to what degree they have read the content of the website and (iv) asking the intervention providers to indicate the extent to which the intervention was delivered as planned.

\section{CONCLUSION}

The present study is the first to describe the planned process for the development, implementation, and process and effect evaluation of a web-based brief alcohol intervention based upon the IM approach. Strengths of IM encompass that WDYD was developed on the basis of theory and empirical evidence enabling other programme planners to identify and retain the crucial elements in order to translate the intervention to new populations and/or settings. Moreover, WDYD is tailored to the target population as a result of IM, which should improve the likelihood of effectiveness of the intervention because less redundant information is given, users' confidentiality is ensured, and the stages of the behavioural change process are considered (Brug et al., 2005). Finally, the use of IM ensured the participation and involvement of the programme planners through all the phases of the project (Fernández et al., 2005), thereby enhancing further collaboration among the programme planners.

Several limitations need to be acknowledged regarding the use of IM. First, the usefulness of IM in the development of web-based brief interventions has not yet been demonstrated. Therefore, it is difficult to determine the successful application of IM in the field of alcohol prevention. Secondly, the iterative planning process of IM might hamper the acquisition of funding, as the intervention is subject to ongoing evaluation and adaptation (Reinaerts et al., 2008). However, this affords flexible and corrective decision-making during the process, eventually resulting in a state-of-art intervention. Third, IM is a time-consuming process. However, it ensured a systematic evidencebased and theory-driven development and implementation of the WDYD intervention. 
We hope to encourage developers in the field of web-based interventions to make the development and implementation of the intervention more transparent in order to understand the underlying mechanisms of the effectiveness of interventions.

\section{FUNDING}

This work was supported by The Netherlands Organization for Health Research and Development (ZonMw) (50-50110-96-682).

\section{REFERENCES}

Ajzen, I. (1991) The theory of planned behavior. Organizational Behavior and Human Decision Processes, 50, 179-211.

Anderson, P., de Bruijn, A., Angus, K., Gordon, R. and Hastings, G. (2009) Impact of alcohol advertising and media exposure on adolescent alcohol use: a systematic review of longitudinal studies. Alcohol and Alcoholism, 44, 229-243.

Bandura, A. (1986) Social Foundations of Thought and Action: A Social Cognitive Theory. Prentice-Hall, EnglewoodCliffs, NJ.

Bartholomew, K., Parcel, G., Kok, G. S. and Gottlieb, N. (2001) Intervention Mapping: Designing Theory- and Evidence-Based Health Promotion Programs. Mayfield, Mountain View, CA.

Bewick, B. M., Trusler, K., Barkham, M., Hill, A. J., Cahill, J. and Mulhern, B. (2008) The effectiveness of web-based interventions designed to decrease alcohol consumption - a systematic review. Preventive Medicine, 47, 17-26.

Bodenheimer, T. and Handley, M. A. (2009) Goal-setting for behavior change in primary care: an exploration and status report. Patient Education and Counseling, 76, $174-180$.

Borsari, B. and Carey, K. B. (2000) Effects of brief motivational intervention with college student drinkers. Journal of Consultancy and Clinical Psychology, 68, 28-33.

Borsari, B., Murphy, J. G. and Barnett, N. P. (2007) Predictors of alcohol use during the first year of college: Implications for prevention. Addictive Behaviors, 32, 2062-2086.

Brug, J., Oenema, A. and Ferreira, I. (2005) Theory, evidence and Intervention Mapping to improve behavior nutrition and physical activity interventions. International Journal of Behavioral Nutrition and Physical Activity, 2, 2.

CBS. (2010) Gezondheid, leefstijl, gebruik van zorg [Health, lifestyle, care use] CBS: Statistics Netherlands.

Collins, S. E. and Carey, K. B. (2007) The theory of planned behavior as a model of heavy episodic drinking among college students. Psychology of Addictive Behaviors, 21, 498-507.
Cullum, J., Armeli, S. and Tennen, H. (2010) Drinking norm-behavior association over time using retrospective and daily measures. Journal of Studies on Alcohol and Drugs, 71, 769-777.

Danielsson, A. K., Wennberg, P., Tengstrom, A. and Romelsjo, A. (2010) Adolescent alcohol use trajectories: predictors and subsequent problems. Addictive Behaviors, 35, 848-852.

De Vries, H. and Brug, J. (1999a) Computer-tailored health interventions delivered over the web: review and analysis of key components. Patient Education and Counseling, 74, 156-173.

De Vries, H. and Brug, J. (1999b) Computer-tailored interventions motivating people to adopt health promoting behaviors: introduction to a new approach. Patient Education and Counseling, 36, 99-105.

De Vries, H., Mudde, A., Leijs, I., Charlton, A., Vartiainen, E., Buijs, G. et al. (2003) The European Smoking Prevention Framework Approach (EFSA): an example of integral prevention. Health Education Research, 18, 611-626.

Doumas, D. M., McKinley, L. L. and Book, P. (2009) Evaluation of two web-based alcohol interventions for mandated college students. Journal of Substance Abuse Treatment, 36, 65-74.

Fernández, M. A., Gonzales, A., Tortolero-Luna, G., Partida, S. and Bartholomew, L. K. (2005) Using Intervention Mapping to develop a breast and cervical cancer screening program for Hispanic farmworkers: Cultivando La Salud. Health Promotion Practice, 6, 394-404.

Gezondheidsraad. (2006) Richtlijnen voor gezonde voeding 2006 [Guidelines for healthy nutrition 2006]. Gezondheidsraad. Dutch National Health Council, Den Haag.

Green, L. W. and Kreuter, M. W. (1991) Health Promotion Planning: An Educational and Environmental Approach. Mayfield Publishers, Mountain View, CA.

Greenbaum, P. E., Del Boca, F. K., Wang, C. and Goldman, M. S. (2005) Variation in the drinking trajectories of freshman college students. Journal of Consulting and Clinical Psychology, 73, 229-238.

Hallett, J., Maycock, B., Kypri, K., Howat, P. and McManus, A. (2009) Development of a web-based alcohol intervention for university students: processes and challenges. Drug and Alcohol Review, 28, 31-39.

Hallett, J., Howat, P. M., Maycock, B. R., McManus, A., Kypri, K. and Dhaliwal, S. S. (2012) Undergraduate student drinking and related harms at an Australian university: web-based survey of a large random sample. BMC Public Health, 12, 37. 10.1186/1471-2458-12-37.

Ham, L. S. and Hope, D. A. (2003) College students and problematic drinking: a review of the literature. Clinical Psychology Review, 23, 719-759.

Hibell, B., Andersson, B., Bjarnasson, T., Ahlström, S., Balakivera, O., Kokkevi, A. and Morgan, M. (2004) The ESPAD Report 2003. Alcohol and other Drug Use among Students in 35 European Countries. The Swedish Council for Information on Alcohol and other Drugs, Stockholm.

Hibell, B., Guttormsson, U., Ahlström, S., Balakireva, O., Bjarnason, T., Kokkevi, A. and Kraus, L. (2009) The 2007 ESPAD Report. Substance Use among Students in 35 European Countries. The Swedish Council for Information on Alcohol and other Drugs, Stockholm. 
Hingson, R., Heeren, T., Zakocs, R., Winter, M. and Wechsler, H. (2003) Age of first intoxication, heavy drinking, driving after drinking and risk of unintentional injury among U.S. college students. Journal of Studies on Alcohol and Drugs, 64, 23-31.

Janz, N. K. and Becker, M. H. (1984) The Health Belief Model: a decade later. Health Education Quarterly, 11, $1-47$.

Ji, C. Y., Hu, P. J. and Song, Y. (2012) The epidemiology of alcohol consumption and misuse among Chinese college students. Alcohol and Alcoholism, 00, 1-9.

Jones, B. T., Corbin, W. and Fromme, K. (2001) A review of expectancy theory and alcohol consumption. Addiction, 96, 57-72.

Kok, G., De Vries, H., Mudde, A. N. and Strecher, V. J. (1991) Planned health-education and the role of selfefficacy-Dutch research. Health Education Research, 6, 231-238.

Kwak, L., Kremers, S. P. J., Werkman, A., Visscher, T. L. S., van Baak, M. A. and Brug, J. (2006) The NHF-NRG In Balance-project: the application of Intervention Mapping in the development, implementation and evaluation of weight gain prevention at the worksite. Obesity Reviews, 8, 347-361.

Kypri, K., Saunders, J. B. and Gallagher, S. J. (2003) Acceptability of various brief intervention approaches for hazardous drinking among university students. Alcohol and Alcoholism, 38, 626-628.

Kypri, K., Hallett, J., Howat, P., McManus, A., Maycock, B., Bowe, S. and Horton, N. J. (2009) Randomized controlled trial of proactive web-based alcohol screening and brief intervention for university students. Archives of Internal Medicine, 169, 1508-1514.

Lebo, H. (2010) The UCLA internet report. Surveying the digital future. Year three. UCLA Center for Communication Policy.

Lewis, M. A., Neighbors, C., Geisner, I. M., Lee, C. M., Kilmer, J. R. and Atkins, D. C. (2010) Examining the associations among severity of injunctive drinking norms, alcohol consumption, and alcohol-related negative consequences: the moderating roles of alcohol consumption and identity. Psychology of Addictive Behaviors, 24, 177-189.

Linke, S., McCambridge, J., Khadjesari, Z., Wallace, P. and Murray, E. (2011) Development of a psychologically enhanced interactive online intervention for hazardous drinking. Alcohol and Alcoholism, 43, 669-674.

Lundborg, P. and Lindgren, O. (2002) Risk perceptions and alcohol consumption among young people. Journal of Risk and Uncertainty, 25, 165-183.

Maalsté, N. (2000) Ad Fundum! Een blik in de gevarieerde drinkcultuur van het Nederlandse studentenleven [Ad Fundum! A Gaze in the Variated Drinking Culture of the Dutch Students' Life]. Centrum Verslavings Onderzoek (CVO), Utrecht.

McKinnon, S., O'Rourke, K. and Byrd, T. (2003) Increased risk of alcohol abuse among college students living on the US-Mexico border: implications for prevention. Journal of American College Health, 51, 163-167.

Miller, W. R. and Rollnick, S. (2002) Motivational Interviewing: Preparing People for Change. Guilford Press, New York.

Murphy, J. G., Correia, C. J. and Barnett, N. P. (2007) Behavioral economic approaches to reduce college student drinking. Addictive Behaviors, 32, 2573-2585.
Neighbors, C., Larimer, M. E. and Lewis, M. A. (2004) Targeting misperceptions of descriptive drinking norms: efficacy of a computer-delivered personalized normative feedback intervention. Journal of Consulting and Clinical Psychology, 72, 434-447.

Norstrom, T. and Ramstedt, M. (2005) Mortality and population drinking: a review of the literature. Drug and Alcohol Review, 24, 537-547.

Oei, T. P. S. and Morawska, A. (2004) A cognitive model of binge drinking: the influence of alcohol expectancies and drinking refusal self-efficacy. Addictive Behaviors, 29, 159-179.

Oei, T. P. S., Hasking, P. A. and Young, R. M. (2005) Drinking refusal self-efficacy questionnaire-revised (DRSEQ-R): a new factor structure with confirmatory factor analysis. Drug and Alcohol Dependence, 78, 297-307.

O'Neill, S. E., Parra, G. R. and Sher, K. J. (2001) Clinical relevance of heavy drinking during the college years: cross-sectional and prospective perspectives. Psychology of Addictive Behaviors, 15, 350-359.

Poelen, E. A. P., Scholte, R. H. J., Engels, R. C. M. E., Boomsma, D. I. and Willemsen, G. (2005) Prevalence and trends of alcohol use and misuse among adolescents and young adults in the Netherlands from 1993 to 2000. Drug and Alcohol Dependence, 79, 413-421.

Prochaska, J. O. and Velicer, W. F. (1997) The transtheoretical model of health behavior change. American Journal of Health Promotion, 12, 38-48.

Ragsdale, K., Porter, J. R., Mathews, R., White, A., Gore-Felton, C. and Mcgarvey, E. L. (2011) 'Liquor before beer, you're in the clear': binge drinking and other risk behaviours among fraternity/sorority members and their non-Greek peers. Journal of Substance Use, 17, 323-339.

Rehm, J., Mathers, C., Popova, S., Thavorncharoensap, M., Teerawattananon, Y. and Jayadeep, J. (2009) Global burden of disease and injury and economic cost attributable to alcohol use and alcohol-use disorders. The Lancet, 373, 2223-2233.

Reinaerts, E., De Nooijer, J. and De Vries, N. K. (2008) Using Intervention Mapping for systematic development of two school-based interventions aimed at increasing children's fruit and vegetable intake. Health Education Monographs, 108, 301-320.

Riper, H., Kramer, J., Smit, F., Conijn, B., Schippers, G. and Cuijpers, P. (2008) Web-based self-help for problem drinkers: a pragmatic randomized trial. Addiction, 103, 218-227.

Riper, H., van Straten, A., Keuken, M., Smit, F., Schippers, G. and Cuijpers, P. (2009) Curbing problem drinking with personalized-feedback interventions: a meta-analysis. American Journal of Preventive Medicine, 36, 247-255.

Spijkerman, R., Roek, M. A. E., Vermulst, A., Lemmers, L., Huiberts, A. and Engels, R. C. M. E. (2010) Effectiveness of a web-based brief alcohol intervention and added value of normative feedback in reducing underage drinking: a randomized controlled trial. Journal of Medical Internet Research, 12, e65.

Steckler, A. and Linnan, L. (2002) Process Evaluation for Public Health Interventions and Research. Jossey-Bass Publishers, San Francisco, CA.

Stone, A. A. and Shiffman, S. (1994) Ecological momentary assessment in behavioural medicine. Annals of Behavioral Medicine, 16, 199-202. 
Tortelo, S. R., Markham, C. M., Parcel, G. S., Peters, R. J., Escobar-Chaves, S. L., Basen-Engquist, K. et al. (2005) Using Intervention Mapping to adapt an effective HIV, sexually transmitted disease, and pregnancy prevention program for high-risk minority youth. Health Promotion Practice, 6, 286-298.

Tripp, M. K., Herrmann, N. B., Parcel, G. S., Chamberlain, R. M. and Gritz, E. R. (2000) Sun protection is fun! A skin cancer prevention program for preschools. Journal of School Health, 70, 395-401.

Turrisi, R., Larimer, M. E., Mallett, K. A., Kilmer, J. R., Ray, A. E., Mastroleo, N. R. et al. (2009) A randomized clinical trial evaluating a combined alcohol intervention for high-risk college students. Journal of Studies on Alcohol and Drugs, 70, 555-567.

Van Empelen, P., Kok, G., Schaalma, H. P. and Bartholomew, L. K. (2003) An AIDS risk reduction program for Dutch drug users: an intervention mapping approach to planning. Health Promotion Practice, 4, $402-412$.

Verster, J. C. (2011) Editorial: US students' alcohol consumption: a downward trend, but increased concerns. Curr Drug Abuse Rev, 4, 208-209.

Voogt, C. V., Poelen, E. A. P., Kleinjan, M., Lemmers, L. A. C. J. and Engels, R. C. M. E. (2011) Targeting young drinkers online: the effectiveness of a web-based brief alcohol intervention in reducing heavy drinking among college students: Study protocol of a two-arm parallel group randomized controlled trial. BMC Public Health, 11, 231.
Wallace, P., Murray, E., McCambridge, J., Khadjesari, Z., White, I. R., Thompson, S. G. et al. (2011) On-line randomized controlled trial of an internet based psychologically enhanced intervention for people with hazardous alcohol consumption. PLoS ONE, 6, 3 .

Webb, T. L., Joseph, J., Yardley, L. and Michie, S. (2010) Using the internet to promote health behavior change: a systematic review and meta-analysis of the impact of theoretical basis, use of behavior change techniques, and mode of delivery on efficacy. Journal of Medical Internet Research, 12, e4.

Wild, T. C., Cunningham, J. A. and Roberts, A. B. (2007) Controlled study of brief personalized assessmentfeedback for drinkers interested in self-help. Addiction, 102, 241-250.

Wood, M. D., Read, J. P., Palfai, T. P. and Stevenson, J. F. (2001) Social influence processes and college student drinking: the mediational role of alcohol outcome expectations. Journal of Studies on Alcohol and Drugs, 62, 32-43.

Young, R. M., Connor, J. P., Ricciardelli, L. A. and Saunders, J. B. (2006) The role of alcohol expectancy and drinking refusal self-efficacy beliefs in university student drinking. Alcohol and Alcoholism, 41, 70-75.

Young, R. M., Hasking, P. A., Oei, T. P. S. and Loveday, W. (2007) Validation of the drinking refusal self-efficacy questionnaire-Revised in an adolescent sample (DRSEQ-RA). Addictive Behaviors, 32, 862-868.

Zeigler, D. W., Wang, C. C., Yoast, R. A., Dickinson, B. D., McCaffree, M. A., Robinowitz, C. B. et al. (2005) The neurocognitive effects of alcohol on adolescents and college students. Preventive Medicine, 40, 23-32. 